\title{
Deconvolution of Closely Spaced Spectral Features with a Microcalorimeter EDS
}

\author{
Hendrix Demers ${ }^{*, * *}$, Raynald Gauvin ${ }^{* *}$, Ian M. Anderson ${ }^{*}$, David C. Joy ${ }^{*}$, and Edward A. Kenik ${ }^{*}$ \\ *Oak Ridge National Laboratory, Metals and Ceramics Division, Oak Ridge, TN 37831 USA \\ *** McGill University, Dept. Mining, Metals and Materials Engineering, Montreal, Quebec, Canada
}

The superior energy resolution of the current generation microcalorimeter energy-dispersive x-ray spectrometer ( $\mu \mathrm{EDS}, 15 \mathrm{eV} \mathrm{FWHM}$ at $1.5 \mathrm{keV}$ ) allows the resolving of closely spaced spectral features that would overlap with conventional semiconductor EDS (cEDS). This is particularly advantageous in the low energy $(<5 \mathrm{keV})$ region of the spectrum, where widely spaced elements may yield closely spaced characteristic lines and absorption edges of the K, L, and M series. Spectra acquired from elemental chromium provide a good test of the effects of the improved low-energy spectral resolution of $\mu$ EDS because the O-K x-ray line $(525 \mathrm{eV})$ and absorption edge (532 eV) fall between the $\mathrm{Cr}-\mathrm{L}_{\alpha}(573 \mathrm{eV})$ and $-\mathrm{L}_{\ell}(500 \mathrm{eV})$ lines. The chromium spectrum is therefore sensitive to the formation of a thin chromium oxide scale, or to the differential absorption of the $\mathrm{Cr}-\mathrm{L}_{\alpha}$ and $-\mathrm{L}_{\ell}$ by oxygen from the silica poly capillary focusing optic or ice formation on the detector window. Note that these spectral features would be entirely overlapping with cEDS.

$\mu$ EDS spectra from a nominally pure chromium standard were acquired with the ORNL low-voltage microprobe (JEOL $6500 \mathrm{~F}$ ), operated at $10 \mathrm{kV}$, and equipped with an EDAX Polaris $\mu$ EDS. The effect of the differential absorption of the $\mathrm{Cr}-\mathrm{L}_{\alpha}$ by oxygen is illustrated in Fig. 1 by chromium spectra acquired: (a) without the poly capillary focusing optic; (b) with the optic but immediately following de-icing; and (c) with the optic after 13 days of ice build-up. Qualitatively, a factor of two decrease is evident in the intensity of the $\mathrm{Cr}-\mathrm{L}_{\alpha}$ relative to the $\mathrm{Cr}-\mathrm{L}_{\ell}$ from (a) to (b) and from (b) to (c). Quantification of x-ray peak intensities were performed by top-hat filtering (filter width $U=$ peak FWHM) and multiple linear least-squares fitting of Gaussian functions as developed for cEDS spectra by Statham [1]. With the spectra binned to $5 \mathrm{eV} /$ channel, the calculated $\mathrm{Cr} \mathrm{L}_{\alpha} / \mathrm{L}_{\ell}$ intensity ratios were (a) 2.06, (b) 0.40 , and (c) 0.24 . The "W" shape of the residual background beneath the fitted peak indicates that a Gaussian function does not adequately describe the shape of the $\mu$ EDS characteristic peaks at this binning. The effect of binning the data on quantitative analysis is demonstrated by re-binning the spectrum in Fig. 1a to $1 \mathrm{eV} /$ channel, Fig. 2. At this finer binning, the calculated FWHMs of the characteristic peaks decrease, the calculated bremsstrahlung backgrounds increase by a factor of $\sim 2$, and the calculated $\mathrm{Cr} \mathrm{L}_{\alpha} / \mathrm{L}_{\ell}$ intensity ratio changes significantly to 2.44. The "W" shape of the residual bremsstrahlung in Fig. 1a is reduced or eliminated in Fig. 2. These studies indicate the importance of sufficient spectral oversampling for quantitative $\mathrm{x}$-ray microanalysis. A plot of $\mathrm{Cr} \mathrm{L}_{\alpha} / \mathrm{L}_{\ell}$ intensity ratio as a function of time, illustrating the absorption effects of both the poly capillary x-ray optic and ice build up is shown in Fig. 3. The strong sensitivity of $x$-ray intensity quantification to these factors indicates the importance of using standards for quantification at the time that microanalysis is performed [2].

\section{References}

[1] Statham, P.J. Analytical Chemistry, 49, (1977), 2149-2154.

[2] Research at the Oak Ridge National Laboratory SHaRE User Facility was sponsored by the Division of Materials Sciences and Engineering, U.S. Department of Energy, under contract DEAC05-00OR22725 with UT-Battelle, LLC. 
a)

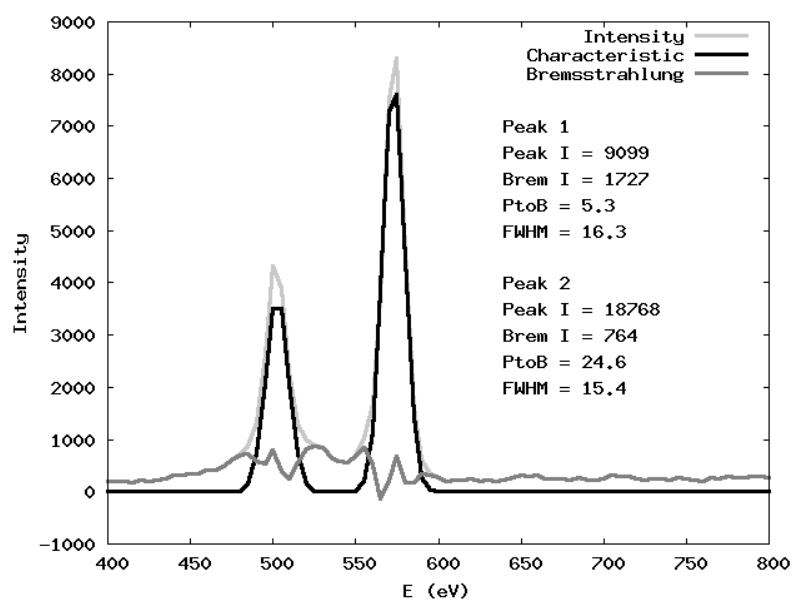

c)
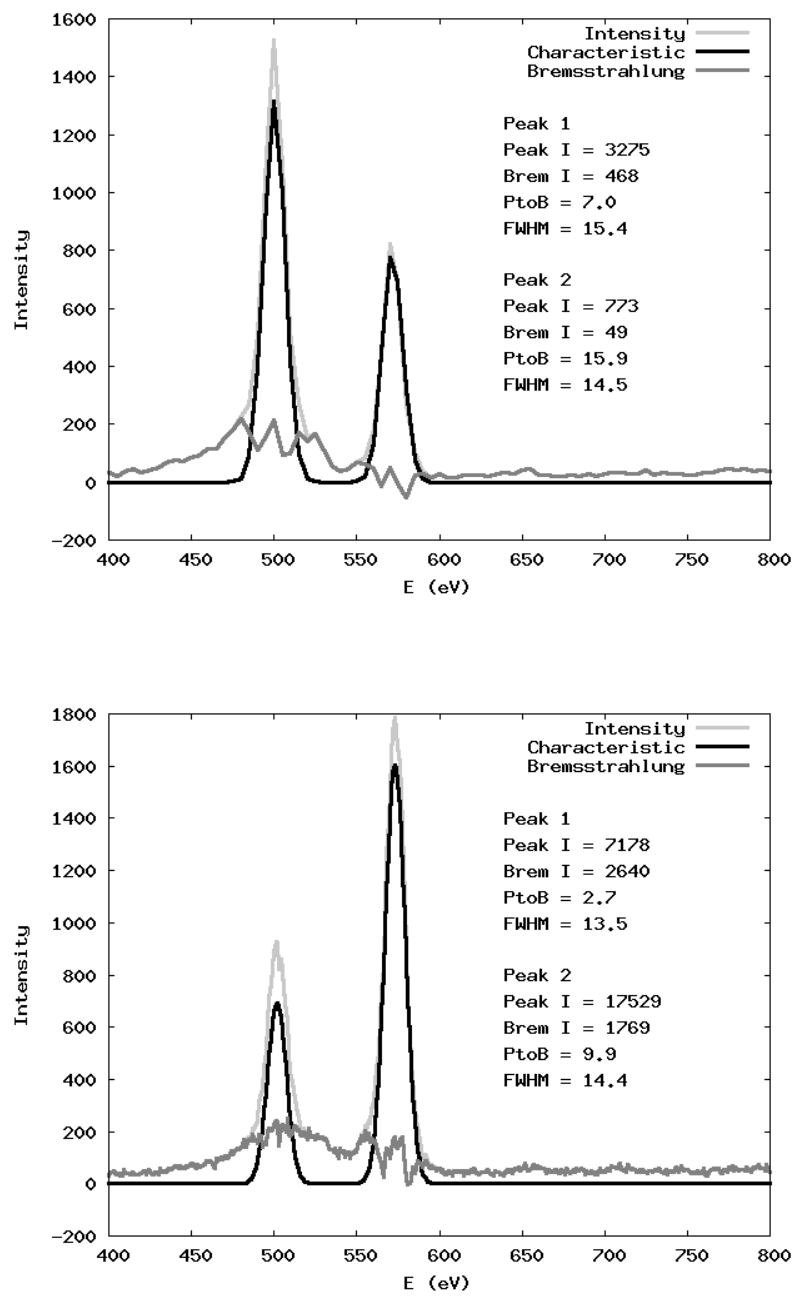

Fig. 2. Chromium $\mu$ EDS spectrum from Fig. 1a displayed and analyzed at $1 \mathrm{eV} \mathrm{/} \mathrm{channel}$ binning.

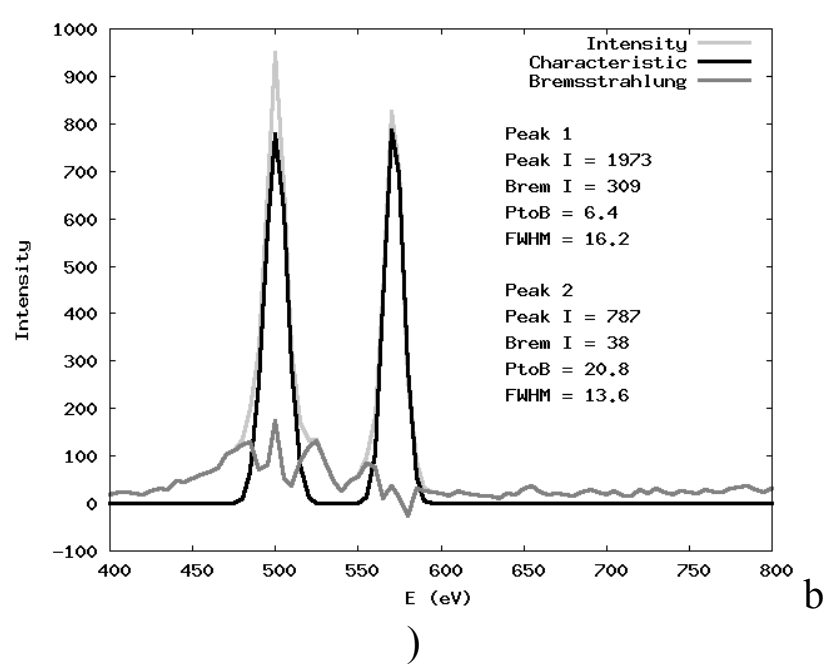

Fig. 1. Chromium $\mu$ EDS spectra binned at $5 \mathrm{eV} /$ channel and $\mathrm{Cr} \mathrm{L}_{\alpha}$ and $\mathrm{L}_{\ell}$ Gaussian peak fitting with background suppression: (a) without x-ray optic; (b) with x-ray optic; and (c) after 13 days of ice build up on the detector window.

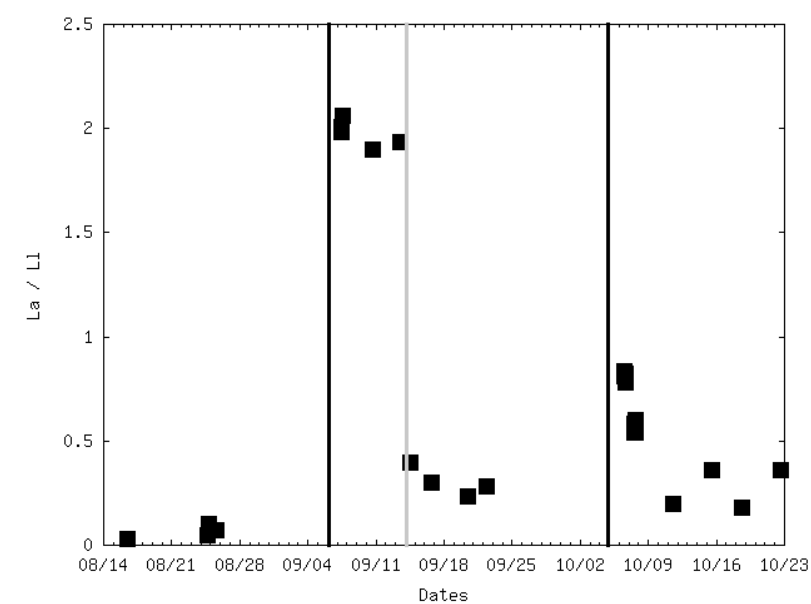

Fig. 3. Plot of $\mathrm{Cr} \mathrm{L}_{\alpha} / \mathrm{L}_{\ell}$ intensity ratio as a function of time, showing the absorption effects of the x-ray optic and ice build up; black lines represent de-icing events and the gray line the installation of the $\mathrm{x}$-ray optic. 